\title{
Docking and molecular dynamics simulations studies of human protein kinase catalytic subunit alpha with antagonist
}

\author{
S. Sandeep, V. Priyadarshini, D. Pradhan, M. Munikumar, A.Umamaheswari \\ Department of Bioinformatics, SVIMS Bioinformatics Centre, Sri Venkateswara Institute of Medical Sciences, Tirupati
}

\begin{abstract}
Background: Cyclic adenosine monophosphate (cAMP) dependent protein kinase A plays major role in cell signalling to undergo many cellular functions. Over expression of extracellular cAMP dependent protein kinase catalytic subunit alpha (PRKACA) causes severe tumorgenesis in prostate. Thus, computer aided high throughput virtual screening and molecular dynamics simulations studies were implemented to identify the potent leads for human PRKACA.

Methods: The human PRKACA crystal structure was optimized in Maestro v9.2. Fifteen recently published PRKACA inhibitors were selected for compiling 5388 structural analogs from Ligand.Info database, these were prepared using LigPrep. Molecular docking from lesser to higher stringency towards minor steric classes was applied subsequently to the prepared ligand dataset into PRKACA active site using Glide v5.7. Molecular dynamics simulation studies were done using Desmond v3.0 to predict the activity of PRKACA-leptosidin complex.

Results: Twenty lead molecules were identified. Lead-1 was observed to have relatively the least docking score compared to the identified lead molecules and 15 published inhibitors. The PRKACA- leptosidin complex deciphered that leptosidin blocked the active site residues Thr-51, Glu-121, Val- 123, Glu-127 and Thr-183 directly through intermolecular hydrogen bonds. In molecular dynamics simulations, trajectory analysis also showed existence of water bridges between PRKACA and leptosidin.

Conclusions: Docking and molecular dynamics studies revealed the better binding interaction of leptosidin with PRKACA. Leptosidin is having the better pharmacological properties thus it could be a futuristic perspective chemical compound for prostate cancer therapy.
\end{abstract}

Keywords: Protein kinase A, cAMP dependent protein kinase catalytic subunit alpha, Prostate cancer, Docking, Molecular dynamics simulations

Supplementary figures available at URL: http://svimstpt.ap.nic.in/jcsr/jhome.htm

Sandeep S, Priyadarshini V, Pradhan D, Munikumar M, Umamaheswari A. Docking and molecular dynamics simulations studies of human protein kinase catalytic subunit alpha with antagonist. J Clin Sci Res 2012;1:15-23.

\begin{tabular}{l}
\hline INTRODUCTION \\
\hline The human genome contains about 500 \\
protein kinase genes. Protein kinases belong \\
to tran ferase family, which get activated \\
through phosphorylation reactions. The \\
activated protein kinases regulate many \\
biological processes like gene expression, \\
signaling mechanisms, cell regulation and \\
extra-intra cellular stimuli. ${ }^{1}$ Mutations, halted \\
cellular inhibition and overexpression or \\
down regulation of protein kinases can result \\
in acute diseases such as cancer, diabetes and \\
deficiency in the immune system. ${ }^{2,3}$ Cancer is \\
a result of tumorgenesis due to genetic and \\
epigenetic alterations which lead to \\
transformation of normal cells into malignant \\
cells. Protein kinases have vital role in
\end{tabular}

tumorgenesis and cell progression, hence, more than 70 kinase inhibitors are under clinical trials against cancer. ${ }^{4}$ Most inhibitors of protein kinases were developed by targeting on the ATP binding site. ${ }^{5-7}$

The cAMP (cyclic adenosine mono phosphate) dependent protein kinase catalytic alpha subunit (PRKACA) of protein kinase A (PKA) belongs to serine/threonine kinase family. The holoenzyme of human PKA has three types of catalytic subunits namely $\mathrm{C} \alpha \mathrm{C} \beta$, and $C \gamma^{8-10}$ and four types of regulatory subunits $\mathrm{RI} \alpha, \mathrm{RI} \beta, \mathrm{RII} \alpha$ and $\mathrm{RII} \beta{ }^{11-}$ ${ }^{14}$ In catalytic core motif, amino cleft involved in Mg-ATP binding site, where as carboxy cleft involved in peptide binding (regulator of C subunit activity) and catalysis. Activation of PKA depends on concentration levels of

Received: 26 December, 2011.

Corresponding author: Dr Amineni Umamaheswari, Associate Professor \& Coordinator of BIF, Department of Bioinformatics, SVIMS Bioinformatics Centre, SVIMS Tirupati 517 507, India. e-mail: svims.btisnet@nic.in 
cAMP. Glucagon receptors bind with the Gproteins and the formed complex coupled with the adenylate cyclase becomes active to further act on ATP for release of cAMP. The released cAMP binds to regulatory subunits and results in free catalytic subunits. The free catalytic subunits can catalyse the transfer of ATP terminal phosphates to protein substrates at serine (or) threonine residues. PKAs are present in a variety of cells, acts on various substrates to participate in metabolism, ${ }^{15}$ gene regulation, ${ }^{16}$ cell growth and division, ${ }^{17}$ cell differentiation, ${ }^{18,19}$ sperm motility ${ }^{20}$ and ion channel conductivity, ${ }^{21}$ among others. Thus, minute alteration in the catalytic subunit of PKA would hamper cell signalling mechanism leading to cancer.

Experimental studies revealed that over expression of extracellular PKA catalytic subunit causes severe tumorgenesis in prostate cancer and other cancers. ${ }^{22,23}$ Therefore, PRKACA is being used as a drug target for various cancers. Quite a few inhibitors of PRKACA are under clinical trials. The present study was carried out for discovery of new class of PRKACA inhibitors with good pharmacokinetic properties using high throughput virtual screening and molecular dynamics simulations.

\section{MATERIAL AND METHODS}

\section{Protein preparation}

Co-crystal structure of human PRKACA with "796", an inhibitor was retrieved from the protein data bank (PDB). ${ }^{24}$ The PRKACA structural features, active site residues and catalytic residues were analyzed using PDBsum $^{25}$. Maestro v9.2 protein preparation wizard was used to fix erroneous atomic representations in crystal structure and its optimization. $^{26}$ The bond orders were assigned to residues of proteins, hydrogen atoms were added and tautomeric states taking place at their normal $\mathrm{pH}$ (7.0) were generated. Protonation states of hydroxyl, histidine groups, "flips" of $\mathrm{C} / \mathrm{N}$ atom and side chain $\mathrm{O} / \mathrm{N}$ atom of the protein were optimized. Impref minimization was carried out using the OPLS (Optimized Potentials for Liquid Simulations) 2005 molecular mechanics force field ${ }^{27}$ with cut off RMSD
(Root Mean Square Deviation) of $0.3 \AA$. A $20 \times 20 \times 20 \AA$ receptor grid $^{28}$ was generated around the active site and catalytic residues of the refined human PRKACA protein using Glide v5.7. ${ }^{29}$

\section{Structural analogs search}

A geometry based analog search for fifteen published inhibitors of PRKACA (BIRB-796, LY333531, MLN-518, SB202190, SB203580, flavopiridol, roscovitine, gleevec, ${ }^{30}$ H89, HA1077, KN62, Mallotoxin, ${ }^{31}$ Balanol, ${ }^{32}$ Go-6983, PP- $2^{33}$ ) was performed in Ligand Info Meta database. Ligand.info provides a user friendly java based tool to find structural analogs of ligands from eight databases (ChemBank, ChemPDB, KEGG Ligand, Anti- HIV NCI (National Cancer Institute), Drug- likeness NCI, Not annotated NCI, AKos GmbH and Asinex Ltd) comprising more than one million small molecules. ${ }^{34,35}$ It can interactively cluster sets of molecules on the user side and automatically download the similar molecules from the server.

\section{Ligand preparation}

LigPrep $^{36}$ is a tool in Schrödinger suite used to generate the 3D structures from 2D representation, searching for tautomers, steric isomers, ionizations states using $\mathrm{Epik}^{37}$ followed by geometry minimization of ligands based upon the OPLS 2005 force field. ${ }^{27}$ The ligands with poor pharmacological properties and reactive func tional groups were discarded applying Lipinski's filter and reactive filter and an inhouse library of non-redundant, pharmacologically preferred conformations were prepared.

\section{Computational docking}

Docking is a procedure to predict the preferable binding orientation between the two molecules to form a stable complex. ${ }^{38}$ Docking calculations were carried out using Glide v5.7. ${ }^{29}$ The prepared and optimized ligands were flexibly docked in the grid box of the protein using MCSA (Monte Carlo based simulated algorithm) based minimization. Glide Score (GScore) was used for representing binding affinity and ranking. Three subsequent docking procedures such as high throughput virtual screening (HTVS), standard precision (SP) and extra precision 
$(\mathrm{XP})^{26,39}$ were implemented to retain lead molecules with better binding affinity in a good binding orientation without steric classes. 10,000 poses were generated during XP docking for each ligand and the best pose was retained after post docking minimization. The molecules which are docked favorably were ranked based on the XP Gscore.

\section{Molecular dynamics}

Molecular dynamics (MD) simulations for docking complex of human PRKACA - lead1 were performed using Desmond $3.2^{40,41}$ as implemented in Schrödinger package with 10 ns (nanoseconds) simulation time. The initial steps of MD simulations were performed by applying OPLS 2005 molecula mechanics force field. ${ }^{27}$ Water molecules were placed to the ligand-receptor complex with simple point charge (SPC) water model. System was neutralized with counter ions, SHAKE algorithm $^{42}$ used to constrain the geometry of water molecules and heavy atom bond lengths with hydrogens, electrostatic interactions applied using particle mesh ewald (PME) method $^{43}$ and periodic boundary conditions (PBC) were used. ${ }^{43}$

The full system of 44,164 atoms was simulated through the multistep MD protocols of Maestro v9.2. Briefly, full system minimization with restraints on solute was performed for maximum 2000 iterations of a hybrid of the steepest descent and the limited memory Broyden - Fletcher - Gold farb-Shanno LBFGS) algorithms, with a convergence threshold of $50.0 \mathrm{kcal} / \mathrm{mol} / \AA^{2}{ }^{2}$ Similar minimization without any restraints was performed with a convergence threshold of 5.0 $\mathrm{kcal} / \mathrm{mol} / \AA{ }^{2}{ }^{2}$ Non hydrogen solute atoms were restrained in the NVT ensemble (constant number of atoms $\mathrm{N}$, volume $\mathrm{V}$ and temperature $\mathrm{T}$ ) using 10 picoseconds (ps) simulation time and temperature of $10 \mathrm{~K}$. Simulations restraining non hydrogens solute atoms were performed in the NPT ensemble (constant number of atoms $\mathrm{N}$, pressure $\mathrm{P}$ and temperature $\mathrm{T}$ ) for $12 \mathrm{ps}$ simulation time and temperature of $10 \mathrm{~K}$. Further, NPT ensemble for a simulation time of 24 ps restraining all nonhydrogen solute atoms (temperature $300 \mathrm{~K}$ ) and NPT ensemble, without restraints, for a simulation time of 24 ps (temperature $300 \mathrm{~K}$ ) was performed to relax the system.

The relaxed system was simulated for a simulation time of 10000 ps with a time step of 2 femtosecond (fs), NPT ensemble using a Berendsen thermostat at $310 \mathrm{~K}$ and velocity resampling for every $1 \mathrm{ps}$. Trajectories after every 4.8 ps were recorded. Energy fluctuations and RMSD of the complex in each trajectory were analyzed with respect to simulation time. The backbone and side chain root mean square fluctuations (RMSF) of each residue PRKACA were monitored for consistency. The inter-molecular interactions of lead1-PRKACA were assessed for stability of the docking complex.

$\overline{\text { RESULTS }}$

\section{Structural analysis of PRKACA}

The co-crystal structure of PRKACA in complex with $796^{24}$ provides novel insight to develop a new class of inhibitor designing. The protein is of 351 residues and comprises of 15 helices and 14 strands (Figure 1A). Asp166, Lys168, Glu170, Asn171 and Thr201 were reported as catalytic residues in catalytic site atlas (CSA) annotation (Figure 1A). The residues such as Leu49, Gly50, Thr51, Gly52, Gly55, Arg56, Ala70, Lys72, Ile73, Leu74, Val 103, Met120, Glu121, Tyr122, Val123, Asn171, Leu173, Thr183, Asp184 and Phe327 were localized to be in van der Waal contact with "796" constitute as the active site residue (Figure 1A). PRKACA residues Leu49, Thr51, Gly52, Gly55, Arg56, Ala70, Lys72, Val104, Met120, Glu121, Tyr122, Val123, Asn171, Leu173, Thr183, Asp184 and Phe327 were involved in hydro phobic interactions with '796' (Figure 1B). The ligand '796' formed four $\mathrm{H}$-bonds with the residues Glu121, Val123, Asn171 (catalytic residue) and Asp184 (Figure 1B). Further, assessments CASTp analysis supported the role of these residues to form binding pocket in human PRKACA.

\section{Ligand dataset optimization}

Compounds with similar structural geometry would deliver the similar effects; $;^{29,30}$ therefore, instead of implementing docking method to 
small molecule databases directly, it is better to implement docking methods to a set of structural analogs for published inhibitors for identification of new class of inhibitors. Simplified molecular input line entry (SMILE) specification formats of 15. PRKACA published inhibitors were imported in to Ligand.info tool and 5388 structural analogues were obtained. The structural analogues were converted to their 3D structural forms. Multiple conformation generation of each analogue (3D structure) was produced with 20494 ligand conformers. The conformations with higher energetic state, not following Lipinski's rule of five and include reactive functional groups were discarded from the dataset and 7470 non-redundant pharmacologically preferred conformations were retained for virtual screening.

\section{Virtual screening}

One of the most widely used methods for high throughput virtual screening is docking of small molecules into active site of protein target and subsequent scoring. A wide range of different docking programs are available, most of which use semi-rigid docking, where the ligands are treated as flexible and the receptors as rigid. Glide v5.7 is one of the efficient docking programs widely used for fast and extremely accurate binding mode predictions of ligands with protein. Maestro v9.2 virtual screening workflow implements three modes of Glide docking to make the new class of inhibitor searching procedure fast and efficient. The docking modes penalizes lesser to higher towards minor steric classes from HTVS to XP docking. Each docking mode involves Gscore for representing binding affinity and ranking ligands. The Glide HTVS mode of docking was performed to 7470 conformers and ranked. Top 747 ligands (10\%) were selected for subsequent SP docking and ranked based on SPG Score. The top 75 compounds were redocked using Glide XP docking method. Top ranked compounds obtained through XP docking were compared favorably with XPG score of 15 published inhibitors (Supplementary Figure 1). Among published inhibitors, balanol and '796' have showed the lowest XPG score of $-10.63 \mathrm{Kcal} / \mathrm{mol}$. Lead ' 1 '

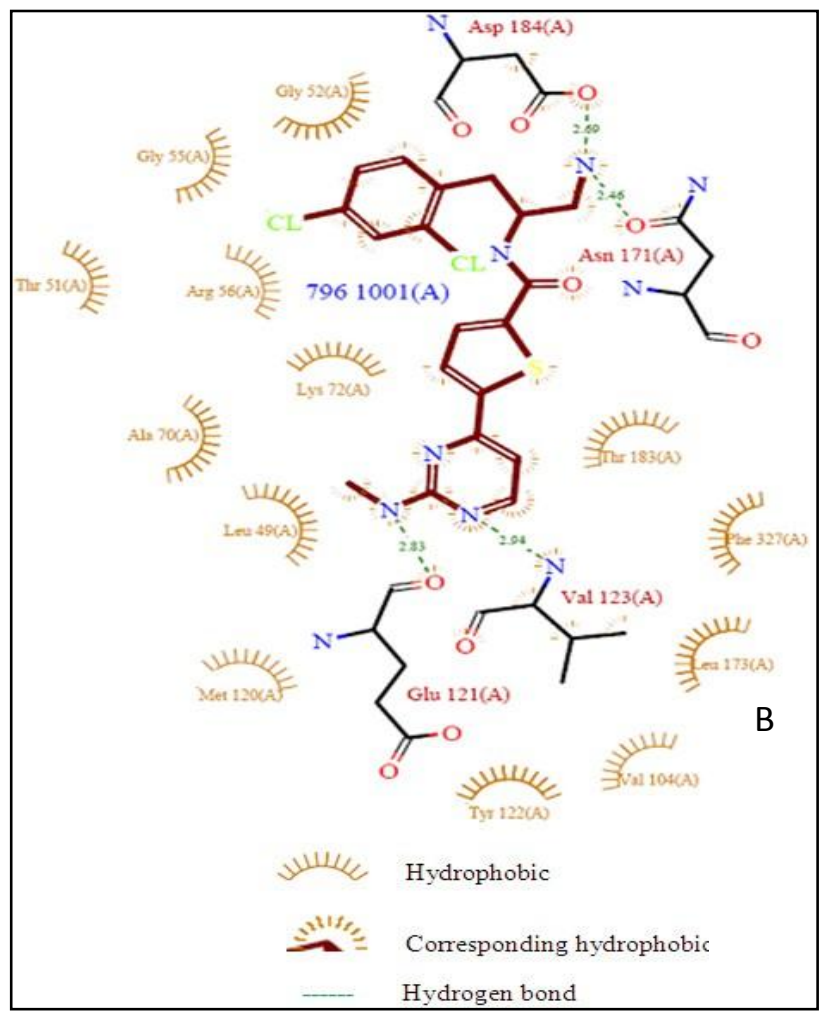

Figure 1: Structural features and active site residues of PRKACA-'796' Complex. A. Secondary structural elements, catalytic residues and ligand contact residues of PRKACA. B. Hydrogen bonding patterns and hydrophobic nteractions of '796' with PRKACA 
(leptosidin) was found to have better XPG score than both balanol and hence '796' has better binding affinity compared to the existing PRKACA inhibitors.

\section{PRKACA - leptosidin docking complex}

The virtual screening result showed that, lept sidin (Supplementary Figure 2) had the lowest XPG score of $-11.02 \mathrm{Kcal} / \mathrm{mol}$ (Supplementary Figure 1) compared to proposed leads and 15 published inhibitors. The good binding affinity of leptosidin is due to various interactions such as hydrogen bonding, hydrophobic interactions, hydrophilic interact tions, electrostatic interactions and steric inter actions. The PRKACA-leptosidin docked complex was visualized in Figure $2 \mathrm{~A}$ and with residues involved in inter-atomic contact was shown in Figure 2B.

Leptosidin was bound to PRKACA with five $\mathrm{H}$ (hydrogen)-bonds in addition to hydrophobic and van der Waal (vdW) interactions (Figure 2BThe two $\mathrm{H}$-atoms $(17(\mathrm{O})-\mathrm{H}$ and $19(\mathrm{O})-\mathrm{H})$ of hydroxyl group of leptosidin catechol ring formed $\mathrm{H}$-bonds with backbone atom of polar residue, Thr51 $(1.778 \AA)$ and side chain atom of charged Glu127 (1.393 $\AA$ ). The hydroxyl group $(5(\mathrm{O})-\mathrm{H})$ of leptosidin guaiacol ring formed two H-bonds with carboxyl group of Glu121 $(2.304 \AA)$ and hydrophobic group of Val123
$(2.02 \AA)$ Oxygen atom $(10(\mathrm{O}))$ of leptosidin formed one $\mathrm{H}$-bond with polar side chain hydrogen of Thr183 (2.439 ̊). The residues Leu49, Gly50, Thr51, Gly52, Gly55, Arg56, Ala70, Lys72, Ile73, Leu74, Val103, Met120, Glu121, Tyr122, Val123, Asn171, Leu173, Thr183, Asp184 and Phe327 and Tyr336 were involved with good van der Waal interactions with leptosidin.

Pharmacokinetic properties such as molecular weight, hydrogen bond donors, hydrogen bond acceptors, $\log \mathrm{P}$ (water/octanol), water partition coefficient $(\mathrm{QP} \log \mathrm{Po} / \mathrm{w})$, water solubility (QPlogS), permeability through Madin-Darby Canine Kidney Cells (QPlogMDCK), Qik Prop predicted log IC50 value for blockage of $\mathrm{K}^{+}$channels (QPlogHERG), QikProp predicted gutblood barrier (QPPCaco), violations of the Lipinski's rule of five (LROF), cell permeability (QPPCaco) etc. are critical for estimation of absorption and distribution of drugs within the body, drug metabolism and its access to biological membranes. All these values for leptosidin were well within the acceptable range for a drug with good pharmacological properties (Table 1).

\section{Molecular dynamics simulations}

Receptor structural rearrangements: Stability of PRKACA - leptosidin complex was evaluated through $10 \mathrm{~ns}$ molecular dynamic

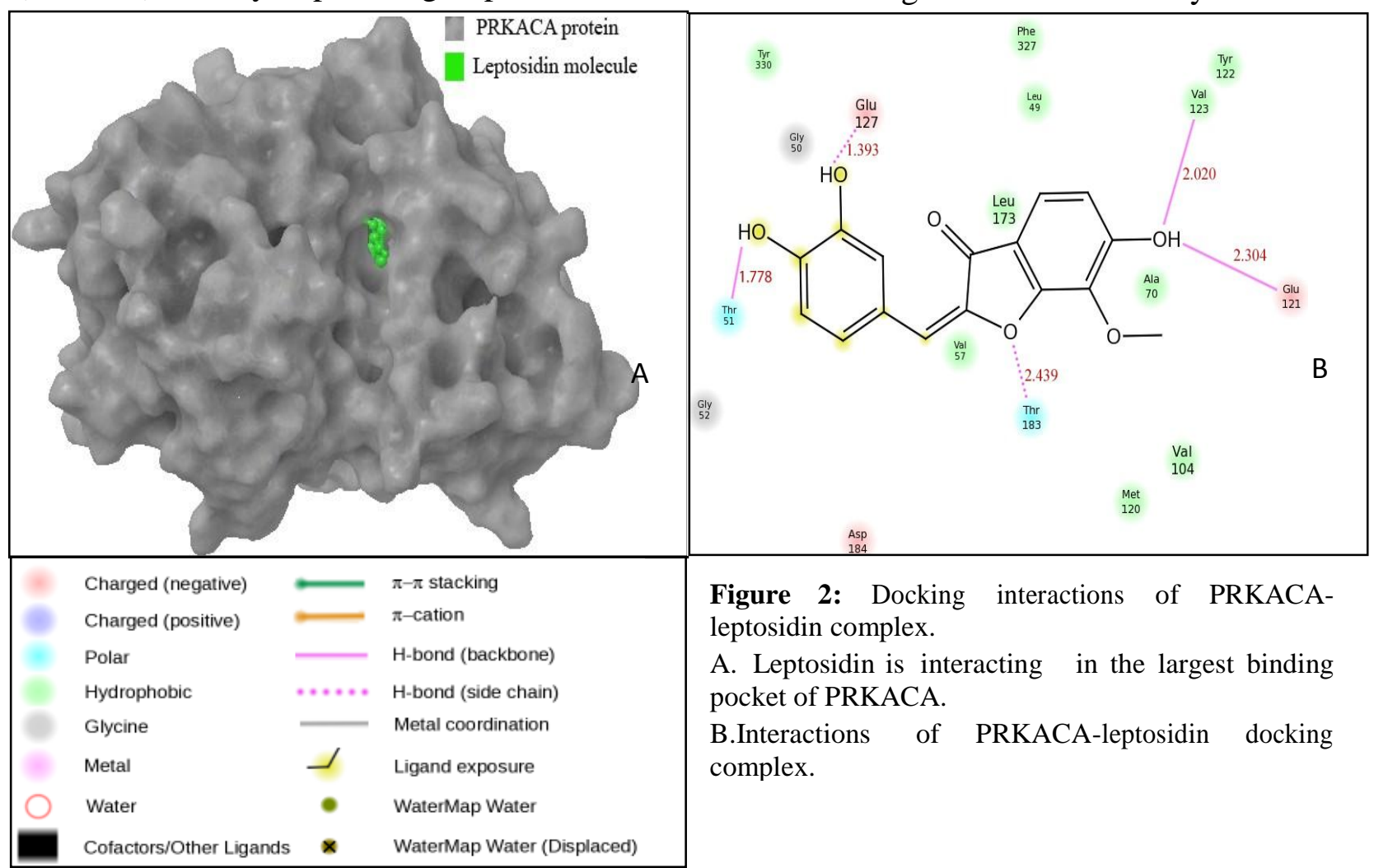


simulations. The simulations provided exact binding interaction of the docking complex with system embedded with water molecules, temperature and pressure. The complex of PRKACA- leptosidin was originated in all proper binding poses with an acceptable RMSD value $(<2 \AA)$. RMSD values of the protein backbone atoms and the heavy atoms of inhibitor during the production phase relative to the starting structures were determined and plotted (Figure 3A).

Table 1: Pharmacokinetic properties of leptosidin

\begin{tabular}{lll}
\hline S.No & $\begin{array}{l}\text { Pharmacokinetic } \\
\text { properties }\end{array}$ & Values \\
\hline 1 & QPlogMDCK & 62.50 \\
2 & QPP Caco & 147.4 \\
3 & QPlogPo/w & 1.381 \\
4 & QPlogS & -3.95 \\
5 & LogPC & 10.316 \\
6 & Oral absorption & 73.847 \\
7 & Ligand Efficiency & -0.501 \\
8 & H-bond donors & 3 \\
9 & H-bond acceptors & 5.5 \\
10 & Molecular weight & 300.267 \\
11 & QPlogHERG & -5.176 \\
12 & LROF & 0 \\
\hline
\end{tabular}

RMSD plot revealed that the complex was relatively stable throughout the simulation time. The overall RMSD range for backbone atoms were $0.6-1.8 \AA$ and heavy atoms were $0.7-2.2 \AA$. The RMSF values of backbone and side chain atoms were assessed for each residue of PRKACA (Figure 3B). Side chain fluctuations of $90 \%$ of residues were observed within the range of $0.5-2.0 \AA$; $94 \%$ of backbone residue fluctuations were within the range of $0.4-1.5$ $\AA$. Side chain of Lys-319 showed a maximum fluctuations of $3 \AA$. Maximum backbone fluctuations up to $3 \AA$ was observed for serine. Inhibitor binding pocket residues showed comparatively lower backbone and side chain fluctuations compared to other residues. The small range of RMSDs and RMSFs reflected slight structural rearrangement in the docking complex during simulation time. The energy plot showed that the energy of the system was relatively consistent during $10 \mathrm{~ns}$ MD simulations run (Figure 3C).

\section{Interactions of PRKACA-leptosidin complex during simulations}

The influence of lead 1 on the dynamical properties of PRKACA active site have been clarified by analyzing the trajectory data obtained from the MD simulations. The PRKACA-leptosidin docking interactions were reproduced during the entire simulations period (Figure 3D). The analysis revealed crucial role of water molecules in PRKACAleptosidin interactions. In most of the trajectories, active site residues of PRKACA were involved in H-bonding through water bridges (Figure 3D). The $\mathrm{H}$ bonds observed for Glu-121 and Val-123 in the PRKACA-leptosidin docking complex remained stable throughout the simulation period (Figures 3D and 3E). Glu-127 was observed to form H-bond with leptosidin till 2000ps simulation time. In some trajectories Glu-127 formed H-bond with the one water bridge (supplementary Figure $3 \mathrm{~A}$ ) while in other trajectories it formed direct $\mathrm{H}$-bonds with leptosidin (supplementary Figures 3B and 3C). Thr-51 was also forming hydrogen bonding during MDsimulations (supplementary Figures 3B and 3F). In none of the trajectories Thr183-leptosidin H-bond was observed. The analysis revealed existence of additional H-bonds between leptosidin and Glu-170 of PRKACA (supplementary Figures $\mathrm{D}, \mathrm{E}$ and $\mathrm{F}$ ). Suitably, Glu-170 was identified as catalytic residue in CSA annotation of PRKACA (Figure1A). Final trajectory of PRKACAleptosidin complex was found to form $8 \mathrm{H}$ bonds (Glu-121, Val-123 directly and Thr-51, Pro-169, Glu-170, Leu-172, Tyr-330 through water bridges (Figure 3D).

In MD simulations, solvating water molecules were considered for checking the stability of ligand-protein complex in contrast to molecular docking. MD simulations were acknowledged closer to the physiological environmental conditions and it reveals better binding conformations for docked complex. The MD simulations for PRKACAleptosidin complex carried out almost reproduced the docking interaction pattern during trajectory analysis. Additionally the analysis revealed that the catalytic residue Glu-170 was blocked by formation of H-bond 
between leptosidin and PRKACA. The RMSD, RMSF, potential energy and interactions of the PRKACAleptosidin complex remained consistent throughout the MD simulations. The complex corroborated well with crystallographic information of PRKACA in complex with inhibitor ARC-1034 and 796.

\section{DISCUSSION}

An elevated level of cAMP dependent protein kinase catalytic subunit alpha in samples of prostate cancer is representing the protein as a drug target for prostate cancer therapy. A systematic virtual screening approach was followed in the present study to propose leptosidin as a potent inhibitor of PRKACA from one million small molecules of Ligand.Info database. The binding modes and interactions of PRKACA-leptosidin docking complex (Figure 2B) were compared with crystallographic data of PRKACA'796'complex (Figures 1A and 1B). Leptosidin is a flavonoid isolated from Coreopsis grandiflora. Favorable comparison of XPG score and binding orientations of leptosidin with the '796' and good pharmacokinetic properties revealed that it was better potential inhibitor for PRKACA than the existing inhibitors.

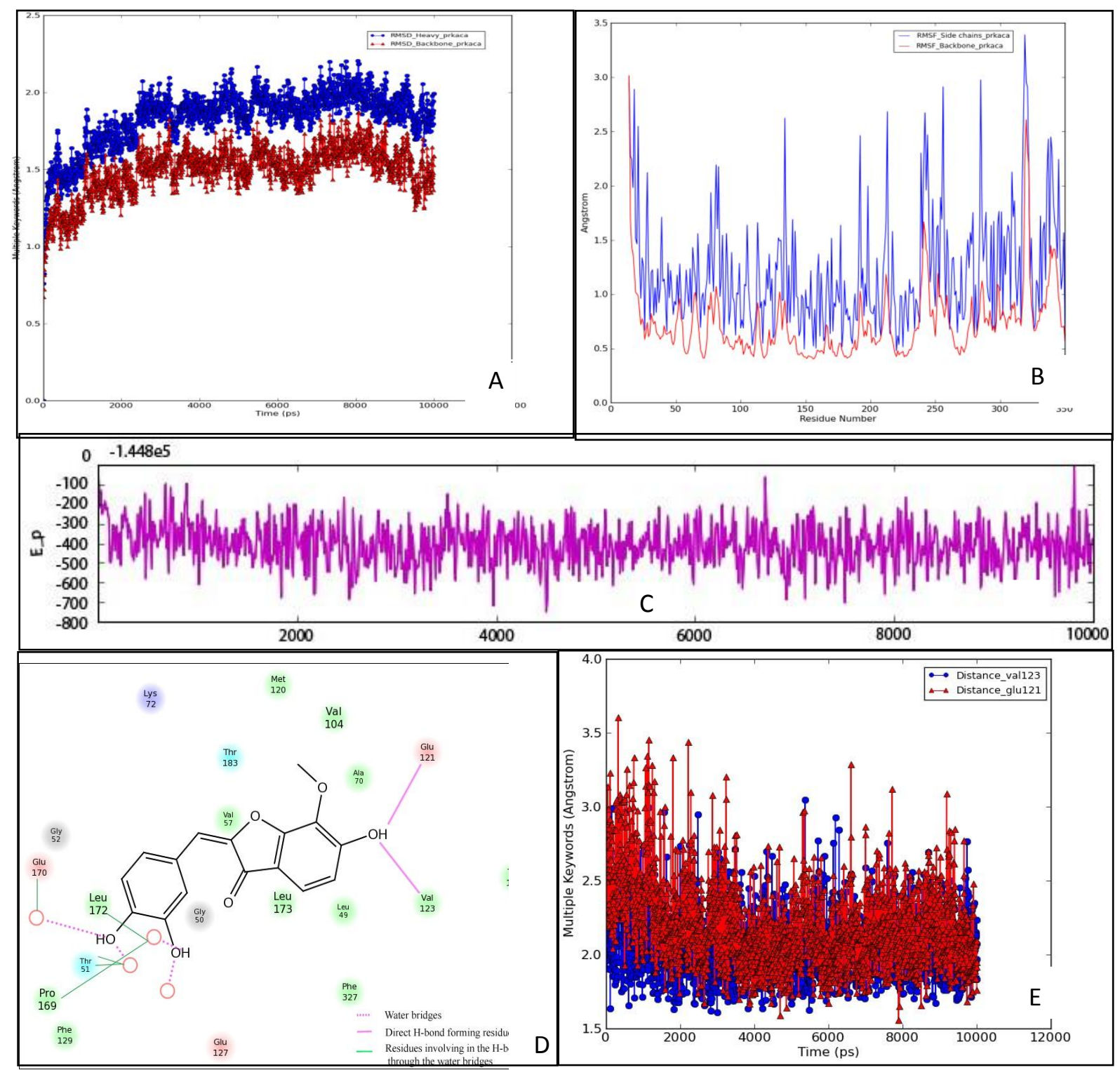

Figure 3: Interactions of PRKACA-leptosidin complex during $10 \mathrm{~ns}$ molecular dynamics simulations run. A. RMSD of heavy atoms and back bone atoms. B. RMSF of PRKACA-leptosidin docked complex. C. Energy peak of the docked complex. D. PRKACA-leptosidin complex in the last trajectory showing $8 \mathrm{H}-$ bonds. E. Hydrogen bond distances of Glu 121 and Val 123. 
Stability of docked complex was further analyzed through the molecular dynamic simulations using Desmond. ${ }^{24,25}$ The docking and MD simulations studies were revealed that the binding affinity of leptosidin towards PRKACA was highly stable with the proposed binding orientations. Analysis of RMSD, RMSF, potential energy and hydrogen bonding interactions of docking complex were rationalized that the steady nature of the lead compound. Active site residues such as Val-123 and Glu-121 residues have formed stable $\mathrm{H}$-bond in all trajectories and complex was consistent (Figure 3D). Additional H-bonds (direct and water bridges) were observed with active site residues such as Leu49, Leu-172, Pro-169, Glu-170 and Tyr-330 during simulation time which revealed the PRKACAleptosidin complex as more stable in the physiological environmental conditions (Figure 3D). Finally, the docked result and MD simulations proved that leptosidin was having better binding orientations, RMSD, RMSF, potential energy and obey good pharmacological properties. Therefore it can be considered for designing drug molecules against prostate cancer.

\section{ACKNOWLEDGEMENTS}

The authors acknowledge the support given by Department of Biotechnology (DBT), Ministry of Science \& Technology, Govt. of India, New Delhi, for research activities at SVIMS Bioinformatics Centre through Biotechnology Bioinformatics Programme.

\section{REFERENCES}

1. Cohen P. Protein kinases-the major drug targets of the twenty first century? Nat Rev Drug Discov2002;1:309-5.

2. Noble ME, Endicott JA, Johnson LN. Protein kinase inhibitors: insights into drug design from structure. Science 2004;303:1800-5.

3. Pelech S. Tracking cell signaling protein expression and phosphorylation by innovative proteomic solutions. Curr Pharm Biotechnol 2004;5:69-77.

4. Gill AL, Verdonk M, Boyle RG, Taylor R. A comparison of physicochemical property profiles of marketed oral drugs and orally bioavailable anticancer protein kinase inhibitors in clinical development. Curr Top Med Chem 2007;7:140822.
5. Enkvist E, Lavogina D, Raidaru G, Vaasa A, Viil I, Lust M, et al. Conjugation of adenosine and hexa-(D- arginine) leads to a nanomolar bisubstrate-analog inhibitor of basophilic protein kinases. J Med Chem 2006;49:7150-9.

6. Nam NH, Lee S, Ye G, Sun G, Parang K. ATPphosphopeptide conjugates as inhibitors of Src tyrosine kinases. Bioorg Med Chem 2004;12:575366.

7. Shen K, Cole PA. Conversion of a tyrosine kinase protein substrate to a high affinity ligand by ATP linkage. J Am Chem Soc 2003;125:16172-3.

8. Uhler MD, Chrivia JC, McKnight GS. Evidence for a second isoform of the catalytic subunit of cAMP-dependent protein kinase. J Biol Chem 1986;261:15360-3.

9. Showers MO, Maurer RA. A cloned bovine cDNA encodes an alternate form of the catalytic subunit of cAMP-dependent protein kinase. J Biol Chem 1986;261:16288-91.

10. Beebe SJ, Qyen O, Sandberg M, Frcysa A, Hansson V, Jahnsen T. Molecular cloning of a tissue specific protein kinase (C gamma) from human testis-representing a third isoform for the catalytic subunit of cAMP dependent protein kinase. Mol Endocrinol 1990;4:465-75.

11. Uhler MD, Carmichael DF, Lee DC, Chivia JC, Krebs EG, McKnight GS. Isolation of cDNA clones coding for the catalytic subunit of mouse cAMP dependent protein kinase. Proc Natl Acad Sci US A 1986;83:1300-4.

12. Uhler MD, Chrivia JC, McKnight GS. Evidence for a second isoform of the catalytic subunit of cAMP dependent protein kinase. J Biol Chem 1986;261:15360 -3.

13. Beebe SJ, Oyen O, Sandberg M, Froysa A, Hansson V, Jahnsen T. Molecular cloning of a tissue specific protein kinase (Cgamma) from human testis representing a third isoform for the catalytic subunit of cAMP dependent protein kinase. Mol Endocrinol 1990;4:465-75.

14. Levy FO, Oyen O, Sandberg M, Tasken K, Eskild W, Hansson V, Jahnsen T. Molecular cloning, complementary deoxyribonucleic acid structure and predicted full length amino acid sequence of the hormone inducible regulatory subunit of 3'-5'-cyclic adenosine monophosphatedependent protein kinase from human testis. Mol Endocrinol 1988;2:1364-73.

15. Krebs EG, Beavo JA. Phosphorylation dephosphorylation of enzymes. Annu Rev Biochem 1979; 48:923-59.

16. Roesler WJ, Vandenbark GR, Hanson RW. Cyclic AMP and the induction of eukaryotic gene transcription. J Biol Chem 1988;263:9063-6.

17. Boynton AK, Whitfield JF. The role of cAMP in cell proliferation: A critical assessment of the evidence. Adv Cyclic Nucleotide Res 1983;15:1934.

18. Liu AY. Differentiation-specific increase of cAMP dependent protein kinase in 3T3-L1 cells. J Biol Chem 1982;257:298-306. 
19. Schwartz DA , Rubin CS. Regulation of cAMP dependent protein kinase subunit levels in Friend erythroleukemic cells,Effects of differentiation and treatment with 8-Br-cAMP and methylisobutyl Xanthine. J Biol Chem 1983;258:777-84.

20. Tash JS, Kakar SS, Means AR. Flagellar motility requires the cAMP dependent phosphorylation of a heat stable NP-40- soluble 56 kda protein, axokininaxokinin. Cell 1984;38: 551-9. 21. Li M, West JW, Numann R, Murphy BJ, Scheuer T, Catterall WA. Convergent regulation of sodium channels by protein kinase $\mathrm{C}$ and cAMPdependent protein kinase. Science 1993;261: 143942.

22. Cvijic ME, Kita T, Shih W, DiPaola RS, Chin KV. Extracellular catalytic subunit activity of the cAMP dependent protein kinase in prostate cancer. Clin Can Res 2000;6:2309-17.

23. Wang H, Li M, Lin W, Wang W, Zhang Z, Rayburn ER, et al. Extracellular activity of cyclic AMP dependent protein kinase as a biomarker for human cancer detection: distribution characteristics in a normal population and cancer patients. Cancer Epidem Biomarker Prev 2007;16:789-95.

24. Lin X, Murray JM, Rico AC, Wang MX, Chu DT, Zhou Y,et al. Discovery of 2pyrimidyl-5- amidothiophenes as potent inhibitors for AKT: synthesis and SAR studies. Bioorg Med Chem Lett 2006;16:4163-8.

25. Laskowski RA, Hutchinson EG, Michie AD, Wallace AC, Jones ML, Thornton JM. PDBsum: a Web- based database of summaries and analyses of all PDB structures. Trends Biochem Sci 1997;22:488-90.

26. Maestro 9.0, versuib 70110, Schrodinger, New York.

27. Kaminski GA, Friesner RA, Tirado-Rives J, Jorgensen WL. Evaluation and reparametrization of the OPLS- AA force field for protein via comparison with accurate quantum chemical calculations on peptides. J Phys Chem B 2001;105:6474-7.

28. Friesner RA, Banks JL, Murphy RB, Halgren TA, Klicic JJ, Mainz DT, et al. Glide: a new approach for rapid, accurate docking and scoring. 1. Method and assessment of docking accuracy. J Med Chem 2004;47:1739-49.

29. Friesner RA, Banks JL, Murphy RB, Halgren TA, Klicic JJ, Mainz DT, et

al. Glide: a new approach for rapid, accurate docking and scoring. 1. Method and assessment of docking accuracy. J Med Chem 2004;47:1739-49.

30. Fabian MA, Biggs WH, Treiber DK, Atteridge

$\mathrm{CE}$, Azimioara MD, Benedetti MG, et al. A small molecule kinase interaction map for clinical kinase inhibitors. Nat Biotechnol 2005;23: 329-36. 31. Wang Z. The peptide microarray-based assay for kinase functionality and inhibition study. Methods Mol Biol 2009;570:329-37.

32. Pande V, Ramos JM, Gago F. The Protein Kinase Inhibitor Balanol: Structure-Activity Relationships and Structure-Based Computational Studies. Anti- Cancer Agents In Medicinal Chemistry 2008; 8:638-5.

33. Kinase selectivity results. Available at URL: http://tools.invitrogen.com/downloads/SelectScree n_Data_193.pdf. Accessed on February 12,2012.

34. Umamaheswari A, Pradhan D, Hemanthkumar M. Identification of potential Leptospira phosphoheptose isomerase inhibitors through virtual high throughput screening. Genomics Proteomics Bioinformatics 2010;8:24-55.

35. Umamaheswari A, Kumar MM, Pradhan D, Marisetty H. Docking studies towards exploring antiviral compounds against envelope protein of yellow fever virus. Interdiscip Sci 2011;3:64-77.

36. Chen IJ, Foloppe NJ. Drug-like bioactive structures and conformational coverage with the LigPrep/ ConfGen suite: comparison to programs MOE and catalyst. J Chem Inf Model 2010;50: 822-39.

37. Shelley JC, Cholleti A, Frye LL, Greenwood JR, Timlin MR, Uchimaya M. Epik: a software program for $\mathrm{pK}(\mathrm{a})$ prediction and protonation state generation for drug-like molecules. J. Comput Aided Mol Des 2007;12:681-1.

38. Lengauer T, Rarey M. Computational methods for biomolecular docking. Curr Opin Struct Biol 1996;6:402-6.

39. Friesner RA, Murphy RB, Repasky MP, Frye LL, Greenwood JR, Halgren TA et al. Extra precision glide: docking and scoring incorporating a model of hydrophobic enclosure for proteinligand complexes. J Med Chem 2006;49:6177-96.

40. Shan Y, Kim ET, Eastwood MP, Dror RO, Seeliger MA, Shaw DE. How does a drug molecule find its target binding site? J Am Chem Soc 2011;133:9181-3.

41. Vilar S, Karpiak J, Berk B, Costanzi S. In silico analysis of the binding of agonists and blockers to the $\beta 2$-adrenergic receptor. J Mol Graph Model 2011;29:809-17.

42. Ryckaert JP, Ciccotti G, Berendsen HJC. Numerical-integration of cartesian equations of motion of a system with constraints-moleculardynamics of Nalkanes. J Comput Phys 1977;23:327-1.

43. Essmann U, Perera L, Berkowitz ML, Darden T, Lee H, Pedersen LG. A smooth particle mesh Ewald method. J Chem Phys 1995;103:8577-3. 